\title{
Centrality of Hirschberg Reflex in Young Emmetropic Population of Pakistan
}

\author{
Zeeshan Kamil $^{1}$, Qirat Qurban ${ }^{2}$, Khalid Mahmood ${ }^{3}$ \\ ${ }^{1-3}$ Khalid Eye Clinic, Nazimabad, Karachi
}

\begin{abstract}
Purpose: To find out the Centrality of Hirschberg reflex in young emmetropic females and to correlate it with asthenopia.

Study Design: Cross-sectional study

Place and Duration of Study: Outpatients department of Khalid Eye Hospital, Karachi, from January to December 2019.

Methods: Six hundred young emmetropic females 10 to 25 years of age were included. We performed the Hirschberg test and Jackson cross cylinder to assess the astigmatism and observed near point of convergence to find out convergence insufficiency.

Results: Out of 600 young emmetropic females only one hundred and ninety had a central Hirschberg corneal reflex along with an astigmatism of 0.25 to 0.50 which was observed in one hundred and sixty five patients. One hundred and twenty five had a receded near point of convergence.
\end{abstract}

Conclusion: Acentrality of Hirschberg can be a cause of asthenopia among young emmetropic females.

Key Words: Asthenopia, Astigmatism, Emmetropia, Hirschberg corneal reflex.

How to Cite this Article: Kamil Z, Qurban Q, Mahmood K, Centrality of Hirschberg Reflex in Young Emmetropic Population of Pakistan. Pak J Ophthalmol. 2021, 37 (1): 126-128.

Doi: 10.36351/pjo.v37i1.1156

\section{INTRODUCTION}

There are a number of methods available for assessing the ocular alignment and can be grouped into four basic types; Hirschberg test, cover tests, dissimilar image test, dissimilar target test. ${ }^{1}$

The Hirschberg light reflex method is based on the contention that $1 \mathrm{~mm}$ of deviation of the corneal light reflection corresponds to approximately $7^{\circ}$, or $15 \Delta$, of ocular deviation of the visual axis. Therefore, a light reflex at the pupillary margin is about $2 \mathrm{~mm}$ from the

Correspondence: Zeeshan Kamil

Department of Outpatients, Khalid Eye Hospital, Karachi

dr.zeeshankamil@yahoo.com

Received: November 2, 2020

Accepted: December 4, 2020 pupillary center (with a 4-mm pupil), which corresponds to $15^{\circ}$, or roughly $30 \Delta$, of deviation. A reflex at the mid-iris region is about $4 \mathrm{~mm}$ from the pupillary center, which is around $30^{\circ}$, or $60 \Delta$, of deviation; similarly, a reflex at the limbus is about $45^{\circ}$,or $90 \Delta$, of deviation. ${ }^{2}$

The angle kappa, which is made by the visual axis and the anatomical pupillary axis of the eye, can have an effect on the corneal light reflex measurements. If the fovea is temporal to the pupillary axis, which is normally the case, the corneal light reflection will be slightly nasal to the center of the cornea. This is termed positive angle kappa and simulates exodeviation. If the fovea is nasal to the pupillary axis, the corneal light reflection will be slightly temporal to the center of the cornea. This is called negative angle kappa and simulates esodeviation. An angle kappa does not affect the cover tests. 
Convergence insufficiency incidence has no major change with growing age up to the age of sixty years but increases significantly thereafter. Augmented visual stress of school work and prolonged periods of reading aggravate symptoms in older children. The most common presentation encountered by a clinician is that of a high school or college student who develops symptoms when unwarranted demands are placed on the visual system during long periods of studying. Lack of sleep, illness, and anxiety are also known to exacerbate the problem. ${ }^{3}$

Rarely, any previous research was done to focus exclusively on emmetropic young female population. The purpose of this observational study was to check the Hirschberg reflex in young emmetropic females and to shed light on the non-refractive causes of ocular discomfort.

\section{METHODS}

This observational study was conducted at Khalid Eye Hospital, Karachi, from January 2019 to December 2019. It included six hundred emmetropic females between the ages of 10 to 25 years having gross visual acuity of $6 / 6$ on Snellen chart. The main complaint in this study group were headache, blurring of vision and double vision while reading. Patients having refractive error, previous history of spectacles, history of excessive screen usage more than five hours a day, late night sleep habits, gross media opacity, or history of glaucoma were excluded from the study. A questionnaire/proforma was designed to record demographics, reason of visit in hospital, Hirschberg test finding, visual acuity, subjective refraction, Jackson cross cylinder test, slit lamp ocular examination, extraocular movement along with near point of convergence and accommodation. Main outcome measures were acentrality of Hirschberg light reflex, hidden minor astigmatism and receding near point of convergence. All recruited patients were informed about the study and study approval was obtained by the Ethical Review Committee. Data was analyzed through SPSS version 20 and frequency for age, and variables were obtained.

\section{RESULTS}

This observational study showed that four hundred ten $(68.3 \%)$ out of six hundred females had non-central corneal light reflex, where as one hundred and ninety $(31.7 \%)$ had a central reflex ( $p$ value $<0.005)$. Out of four hundred ten females having non central reflex three hundred fifty $(85.3 \%)$ had corneal reflex on the nasal side of cornea, where as sixty $(14.7 \%)$ females had corneal reflex in the temporal side of cornea ( $p$ value < 0.005$)$. Astigmatism of 0.25 to 0.50 was observed in one hundred and sixty five (27.5\%) patients. Near point of convergence was more than 20 $\mathrm{mm}$ in one hundred and twenty five $(20.8 \%)$ patients.

\section{DISCUSSION}

Young female patients usually complain of tiredness, headache and blurred vision in the absence of any significant refractive error. The hidden causes of these symptoms in this age group could be convergence insufficiency, forcibly compensated phorias, muscle weakness and minor astigmatism which could be responsible for ocular discomfort in emmetropic population. ${ }^{4,5}$

The miscellany in the reported standards of the convergence insufficiency in literature may be due to disparity in target populations, age groups, and facilities for visual assessment, interpupillary distances and geographical factors affecting the results. Scheiman and colleagues suggested that refractive errors are not the only reason for causing abnormal near point of convergence. There are many other causes like phoria, tropias, injuries, trauma and psychological implications. ${ }^{6,7}$ In this study we observed $20.8 \%$ patients had remote near point of convergence more than $20 \mathrm{~mm}$, which could also be the major contributor of symptoms of asthenopia in this considerably emmetropic study group. It is not improbable that anemia can be casually related to convergence insufficiency in view of the frequency of various degrees of hypochromic anaemia in women of this age group. In many cases the onset of symptoms was a sequel to a hemorrhage or menstrual pathology causing anaemia. These situations must provide additional cause for a latent muscular imbalance to become manifest or for insufficiency to appear where no imbalance previously existed. ${ }^{8}$

Jonejo et al reported central Hirschberg reflex in $17 \%$ of patients and also they reported high prevalence of non central corneal reflex in females. ${ }^{9}$ In our study, central Hirschberg was seen in $31 \%$ of cases although this study included only female patients. Hikmatullah reported high incidence of convergence insufficiency in young females. ${ }^{10}$ Sidra reported high frequency of exophoria in convergence insufficiency patients 
mostly in females. ${ }^{11}$ Another study showed that individuals of 18 to 31 years with visual status of $6 / 9$ to $6 / 6$ found asthenopia in emmetropes. ${ }^{12}$ Saba also reported a relationship between asthenopia and convergence insufficiency. ${ }^{7}$

In latent strabismus especially in exophoria, usual symptoms are headache, aching eyes, intermittent blurring of prints while reading and occasional diplopia. ${ }^{13}$ A study found Astigmatism to be the most frequent cause of asthenopia. Female patients were more prone than male patients to complain of asthenopia, at the same time high school students were more likely than primary school children to complain of asthenopia. ${ }^{14}$

Limitation of this study was that it was a small scale study and not a true reflection of the whole population. However, it has highlighted the various hidden causes of symptoms of asthenopia in young emmetropic females.

\section{CONCLUSION}

Majority (85\%) of non-central corneal reflex population having corneal reflection on the nasal side of the cornea, which also could be the possible cause of difficulty in near work or symptoms of asthenopia.

\section{Ethical Approval}

The study was approved by the Institutional review board/ Ethical review board. (ERL-01-20)

\section{Conflict of Interest}

Authors declared no conflict of interest.

\section{REFERENCES}

1. Helveston E, Moodley A. How to check eye alignment and movement. Community Eye Health, 2019; 32 (107): 55.

2. Coi RY, Kushner BJ. The accuracy of experienced strabismologists using the Hirschberg and Krimsky tests. Ophthalmology, 1998; 105 (7): 1301-1306.

3. Matiullah, Khan B, Durrani J. Associations of convergence insufficiency in patients with myopia of age group 15-25 years. Ophthalmology Update, 2018; 16 (1): 470-473.
4. Wee SW, Moon NJ, Lee WK, et al. Ophthalmological factors influencing visual asthenopia as a result of viewing 3D displays. British Journal of Ophthalmology, 2012; 96: 1391-1394.

5. Cohen Y, Segal O, Barkana Y, Lederman R, Zadok D, Pras E et al. Correlation between asthenopic symptoms and different measurements of convergence and reading comprehension and saccadic fixation eye movements. Optometry - Journal of the American Optometric Association, 2010; 81 (1): 28-34.

6. Scheiman M, Mitchell GL, Cotter S, Cooper J, Kulp $\mathbf{M}$, Rouse $\mathbf{M}$, et al. A randomized clinical trial of treatments for convergence insufficiency in children. Arch Ophthalmol. 2005; 123 (1): 14-24.

7. Akram S, Qasim SMA, Saleemullah, Choudry A. Comparison of convergence insufficiency between different refractive status of the eye. Ophthalmology Pakistan, 2018; 8 (3): 28-32.

8. Manson N. Anaemia as an aetiological factor in convergence insufficiency. Brit J. Ophthal. 1962; 46: 674.

9. Jonejo AY, Hassan MU. Strabismus and its type in children of age 6 to 15 years presenting at a public sector hospital of Karachi. Journal of Dow University of Health Sciences, 2019; 13 (1): 24-29.

10. Hikmatullah. To assess the accommodative insufficiency in patients of convergence insufficiency in age group 5-25 years at Hayatabad medical complex, Peshawar. Ophthalmology Update, 2019; 17 (2): 60-64.

11. Sidra S. Frequency of exophoria among the convergence insufficiency patients. International journal of science: Basic and Applied Research (IJSBAR) 2017; Vol. 36 (3): 65-74.

12. Cross FR. Asthenopia and Ocular Headache. Bristol Med Chir J. 1893; 11 (40): 73-84.

13. Shakeel K, Akram S, Ullah S, Qasim MSA, Arshad A. Association of asthenopia, pre-presbyopia and refractive errors in workers involved in hand crafting. Pak J Ophthalmol. 2018; 34 (3): 201-206.

14. Wajuihian SO. Frequency of asthenopia and its association with refractive errors. Afr Vision Eye Health, 2015; 74 (1): 293.

\section{Author's Designation and Contribution}

Zeeshan Kamil; Consultant Ophthalmologist: Examiner, Manuscript writer.

Qirat Qurban; Consultant Ophthalmologist: Data Collection, Manuscript writer.

Khalid Mahmood; Consultant Ophthalmologist: Data Collection.

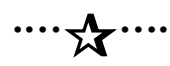

